\title{
Building Biomedical Imaging and Informatics e-Science platform for translational medical research
}

\author{
Jianguo Zhang ${ }^{1 *}$, Kai Zhang ${ }^{1}$, Tusheng Wang ${ }^{1}$, Yuanyuan Yang ${ }^{1}$, Haibo $\mathrm{Hu}^{2}$, Lisa Xu \\ From 2012 Sino-American Symposium on Clinical and Translational Medicine (SAS-CTM) \\ Shanghai, China. 27-29 June 2012
}

\begin{abstract}
Background
As there are urgent demands to bring medical imaging research and clinical service together more closely to solve the problems related to disease discover, medical research, diagnosis and education, a new imaging and informatics infrastructure and paradigm need to be developed to promote multiple disciplines of medical researchers, clinical physicians and biomedical engineers working together in a secured, efficient, and transparent cooperative environment [1]. In this presentation, we outline our preliminary work of building Biomedical Imaging and Informatics (BMII)"e-Science" platform to support collaborative research among multi-disciplines to enable translational research in multiple affiliated hospitals and academic institutions of Shanghai Jiaotong University (SJTU), and Chinese Academy of Sciences (CAS).
\end{abstract}

\section{Materials and methods}

SJTU has 12 large affiliated hospitals located in multiple districts of Shanghai city with a lot of medical and biomedical imaging modalities (e.g., Clinical CT/MR, Micro$\mathrm{PET} / \mathrm{CT}$ ) being decentralized used in these hospitals and research centers. Also, there is a powerful Shanghai Synchronic Radiation Facility (SSRF) developed by CAS to support large scale of biomedical imaging researches from molecular level to organ parts [2]. So, we designed and developed the e-Science platform to promote the multidisciplines working together cross these hospitals and academic institutions, and adopted the Service-Oriented Architecture and grid-based concept to build it. In order

\footnotetext{
* Correspondence: jzhang@mail.sitp.ac.cn

${ }^{1}$ Shanghai Institute of Technical Physics, Chinese Academy of Sciences, Shanghai, China

Full list of author information is available at the end of the article
}

to enable efficient collaborating, we designed the work and data flows with Principal Investigator (PI)-oriented information model, and developed a documents/data sharing mechanism based on IHE XDS/XDS-I profiles and the access control standard of XACML in this platform.

\section{Results}

We implemented the BMII e-Science platform crossing Shanghai Ruijin Hospital, two campuses of SJTU, SSRS and Shanghai Institute of Technical Physics, CAS. The data communications of the e-Science platform from site to site are fast enough as they are going through the China Education Network in Shanghai with backbone of a few of $\mathrm{GB} / \mathrm{sec}$. There were two kinds of collaborations in the eScience platform, one is to perform real-time interactively or synchronously biomedical imaging experiment among onsite users and remote users, and the other is to share the image data or documents among collaborators.

\section{Conclusions}

The developed BMII e-Science platform can promote multiple disciplines of medical researchers, clinical physicians and biomedical engineers working together in a secured, efficient, and transparent cooperative networking environment. Now, the researches, clinical physicians and students can use this e-Science platform to perform biomedical imaging experiments and to do collaborative researching cross multiple hospitals and academic institutions.

\footnotetext{
Acknowledgements

This research are supported by the grant from the Major State Basic Research Development Program of China (973 Program No.: 2010CB834302) and 985 project of Shanghai Jiaotong University.
} 


\section{Author details}

'Shanghai Institute of Technical Physics, Chinese Academy of Sciences, Shanghai, China. ${ }^{2}$ School of Biomedical Engineering and Med-X Research Institute, Shanghai Jiaotong University, Shanghai, China.

Published: 17 October 2012

\section{References}

1. Saltz J, Kurc T, Hastings S, et al: e-Science, caGrid, and Translational Biomedical Research. IEEE Computer 2008, 41:58-66.

2. Xiaoxia Liu, Jun Zhao, Jianqi Sun, Xiang Gu, Tiqiao Xiao, Ping Liu, Lisa X, $\mathrm{Xu}$ : Lung Cancer and Angiogenesis Imaging using Synchrotron Radiation. Physics in Medicine and Biology 2010, 55:2399-2409.

doi:10.1186/1479-5876-10-S2-A32

Cite this article as: Zhang et al:: Building Biomedical Imaging and

Informatics e-Science platform for translational medical research. Journal of Translational Medicine 2012 10(Suppl 2):A32.

Submit your next manuscript to BioMed Central and take full advantage of:

- Convenient online submission

- Thorough peer review

- No space constraints or color figure charges

- Immediate publication on acceptance

- Inclusion in PubMed, CAS, Scopus and Google Scholar

- Research which is freely available for redistribution

Submit your manuscript at www.biomedcentral.com/submit
C Biomed Central 